\title{
Review Article \\ Transcriptional Regulatory Network for the Development of Innate Lymphoid Cells
}

\author{
Chao Zhong and Jinfang Zhu \\ Molecular and Cellular Immunoregulation Unit, Laboratory of Immunology, National Institute of Allergy and Infectious Diseases, \\ National Institutes of Health, Bethesda, MD 20892, USA
}

Correspondence should be addressed to Chao Zhong; zhongc@niaid.nih.gov

Received 12 June 2015; Accepted 22 July 2015

Academic Editor: Timotheus Y. F. Halim

Copyright (c) 2015 C. Zhong and J. Zhu. This is an open access article distributed under the Creative Commons Attribution License, which permits unrestricted use, distribution, and reproduction in any medium, provided the original work is properly cited.

Recent studies on innate lymphoid cells (ILCs) have expanded our knowledge about the innate arm of the immune system. Helperlike ILCs share both the "innate" feature of conventional natural killer (cNK) cells and the "helper" feature of CD4 $4^{+} \mathrm{T}$ helper (Th) cells. With this combination, helper-like ILCs are capable of initiating early immune responses similar to cNK cells, but via secretion of a set of effector cytokines similar to those produced by Th cells. Although many studies have revealed the functional similarity between helper-like ILCs and Th cells, some aspects of ILCs including the development of this lineage remain elusive. It is intriguing that the majority of transcription factors involved in multiple stages of $\mathrm{T}$ cell development, differentiation, and function also play critical roles during ILC development. Regulators such as Id2, GATA-3, Nfil3, TOX, and TCF-1 are expressed and function at various stages of ILC development. In this review, we will summarize the expression and functions of these transcription factors shared by ILCs and Th cells. We will also propose a complex transcriptional regulatory network for the lineage commitment of ILCs.

\section{Introduction}

The mammalian immune system is composed of innate and adaptive arms. T cells and $\mathrm{B}$ cells derived from the lymphoid lineage belong to the adaptive immune system. $\mathrm{CD}^{+} \mathrm{T}$ cells perform a "helper" function via production of effector cytokines after differentiation and activation. In comparison to the "cytotoxic" feature of both adaptive $\mathrm{CD}^{+}{ }^{+} \mathrm{T}$ cells and innate cNK cells, the "helper" feature of $\mathrm{CD}^{+}{ }^{+} \mathrm{Th}$ cells was considered to be a unique characteristic of the adaptive system acquired during evolution. However, over the past few years several groundbreaking works on a novel member of the innate immune system, the innate lymphoid cell (ILC) [1], have dramatically changed our knowledge about the composition of the innate lymphoid lineage [2] and led us to reconsider the relationship between innate and adaptive lymphoid lineages in the context of evolution.

Like other lymphocytes, ILCs also develop from the common lymphoid progenitors (CLPs) found in fetal liver and adult bone marrow. They were not discovered and classified as a new lymphocyte family until recently, partly due to their distinct enrichment in nonlymphoid tissues such as mucosal tissues, skin, and adipose tissues, with scarce distribution in lymphoid tissues. Their lack of any known lineage surface markers may also contribute to their belated discovery. In actuality, scientists noticed certain subsets of ILCs such as lymphoid tissue inducers (LTis) as early as the 1990s [3, 4], but it was not until three independent reports on type 2 cytokine producing innate lymphoid cells (ILC2s) in 2010 [5-7] that people began to recognize the possible existence of an innate population with a "helper" feature mirroring adaptive $\mathrm{Th}$ cells. The nomenclature of innate lymphoid cells (ILCs) was then formed based on the existence of "helper" lymphocyte in the innate arm of the immune system [1].

Similar to the classification of Th cells, mature helperlike ILCs can be categorized into three groups based on their master regulator expression and signature effector cytokine production. ILC2s, the innate counterpart of Th2 cells, express high levels of GATA-3 and are capable of producing type 2 cytokines such as IL-5 and IL-13 [5-7]. ILC3s express ROR $\gamma t$ and are capable of producing IL-22 and IL-17, similar to Th17/Th 22 cells [8-10]. ILC3s can be divided into a CCR6 ${ }^{+}$ lineage and a CCR6 $6^{-}$lineage [11]. The CCR6 ${ }^{+}$lineage includes lymphoid tissue inducer (LTi) cells, while CCR6 ${ }^{-}$ILC3 can 
give rise to a special population of ROR $\gamma \mathrm{t}^{+}$ILC3s that express the natural cytotoxicity receptor (NCR) NKp46 (encoded by Ncrl) in mice and NKp44 (encoded by Ncr2) in humans. These cells are referred to as NCR ${ }^{+}$ILC3s. CCR6 ${ }^{-}$ILC3s can also express T-bet which drives further development of this lineage into the $\mathrm{NCR}^{+}$stage [11-13]. Finally, ILC1s are the innate counterpart of Thl cells. They are T-bet positive and better IFN- $\gamma$ producers than cNK cells $[14,15]$. Absence of ROR $\gamma t$ and Eomes expression in ILCls distinguishes them from ROR $\gamma$ t-expressing $\mathrm{NCR}^{+}$ILC3 and Eomes-expressing cNK cells.

Similar to the relationship between ILCs and Th cells, "cytotoxic" cNK cells represent the innate counterpart of the adaptive cytotoxic $\mathrm{CD}^{+} \mathrm{T}$ cells [16]. Accordingly, both cNK and $\mathrm{CD}^{+} \mathrm{T}$ cells express the transcription factor Eomes. Initially, the nomenclature of ILCs included cNK cells, a notion many scientists still hold. But in this review, we limit ILCs to helper-like ILCs to distinguish these special innate lymphocytes from cNK cells.

\section{Functions of ILCs}

A defining function of ILCs is their production of a similar set of effector cytokines as those produced by $\mathrm{CD} 4^{+} \mathrm{Th}$ cells in the adaptive immune system. This feature of ILCs enables them to mount robust immune responses at the innate stage via acting on other immune or structure cells. Through production of IL-5 and IL-13, ILC2s may induce the first wave of eosinophil recruitment and stimulate epithelial and smooth muscle cells, during type 2 responses to helminth infection or allergen inoculation $[17,18]$. Steady state production of IL22 by ILC3s is crucial for the homeostasis between host and commensals within the mucosal tissues [19]. Upon infection, ILC3s are also the major innate source of IL-22 after receiving IL-23 stimulation $[8,10]$. ILC1 cells are relatively scarce in the gut but enriched in the liver in steady state $[14,20]$. During early type 1 responses, ILC1s are better IFN- $\gamma$ producers than $\mathrm{cNK}$ cells and they provide the initial protection in mice infected with T. gondii [14]. Interestingly, ILCls are also much more potent TNF $\alpha$ producers compared to cNK cells.

Some ILCs can directly interact with Th cells. ILC2s and Th2 cells may collaborate to mount robust type 2 immune responses during the effector phase. Some ILC2s express MHC class II and thus are able to stimulate Th2 cells to produce IL-2, which in turn promotes ILC2 proliferation and cytokine production $[21,22]$. Some ILC3s, mainly within the $\mathrm{CCR6}^{+}$lineage, also express MHC class II [23]. $\mathrm{MHCII}^{+}$ ILC3s can directly present antigen peptide to $\mathrm{CD} 4^{+} \mathrm{T}$ cells. However, possibly due to the lack or low level of costimulatory molecules CD80/CD86 on ILC3s, this type of antigen presentation functions through a suppressive mechanism to maintain the homeostasis of commensal specific Th cell in the colon [24].

ILCs have additional functions, which may or may not be shared by Th cells. For example, ILC2 can produce amphiregulin, which facilitates the repair and reorganization of damaged tissues after viral infection [25]. ILCs are also involved in regulating metabolism. For example, ILC2s are enriched in adipose tissues and contribute to the beiging of white adipose tissue through production of cytokines and/or methionine-enkephalin [26, 27]. Through IL-22 and lymphotoxin production, ILC3s can induce intestinal epithelial cell expression of fucosyltransferase 2 (Fut2) and thus regulate the epithelial fucosylation, which provides the metabolic substrates for commensals $[28,29]$.

\section{Specific Regulators for Distinct ILC Subsets}

The development, maturation, and maintenance of distinct ILC subsets are regulated by a set of specific transcriptional regulators, including T-bet, GATA-3, and ROR $\gamma \mathrm{t}$, similar to the regulation of effector Th cell differentiation. A master regulator that determines Th cell differentiation towards a particular subset also seems to direct the development of the related ILC subset. In addition, other factors such as ROR $\alpha$, $\mathrm{Bcll1b}$, and Ahr are involved in regulating the development and functions of ILC subsets.

3.1. T-bet, GATA-3, and ROR $\gamma t$. Mirroring their critical functions during Th1, Th2, and Th17 differentiation, the master regulators T-bet, GATA-3, and ROR $\gamma$ t are also implicated in fate determination of ILC subsets. GATA-3 is expressed at much higher levels in ILC2s compared with other ILC subsets. Gata3 deficiency during any stages of ILC2 development will eliminate this lineage. Furthermore, GATA-3 is required for the maintenance and function of fully developed ILC2s [30, 31]. Deletion of Gata3 in mature ILC2s results in dramatic diminution of IL-5 and IL-13 production followed by the rapid disappearance of these cells. GATA-3 is also expressed in ILC1 and ILC3 cells as well as in ILC progenitors, in which GATA-3 has a critical function; we will discuss GATA-3 function during early ILC development in detail in the progenitor section below.

ROR $\gamma t$ is uniquely expressed by ILC3s in both CCR $6^{+}$ and CCR6 $6^{-}$subsets. ROR $\gamma t$ deficiency results in a complete loss of ILC3s but not ILC1s or ILC2s. Some CCR6 ${ }^{-}$ILC3s also express T-bet [11]. T-bet induction in ILC3s may be driven by Notch signals [12], IL-23 stimulation, and the microbiota [11]. The gradient increment of T-bet levels directs further development of CCR6 ${ }^{-}$ILC3s into $\mathrm{NCR}^{+}$ILC3s. Some cells may even turn off ROR $\gamma$ t expression to become T-bet ${ }^{+} \mathrm{NCR}^{\text {hi }}$ ex-ILC3s. In addition, T-bet regulates IFN- $\gamma$ production by the $\mathrm{NCR}^{+}$ILC3s [11].

Besides CCR6 ILC3s, ILC1s also express T-bet. Accordingly, T-bet deficient mice lack ILC1s and $\mathrm{NCR}^{+}$ILC3s but have normal ILC2s and CCR6 ${ }^{+}$ILC3s. ILC1s were previously confused with cNK cells as both express T-bet and are capable of producing IFN- $\gamma$. However, cNK cells express Eomes while ILC1s do not. Therefore, Eomes is a good marker to distinguish cNK cells from ILC1s. In addition, ILC1s may also express cell surface markers such as CD49a [14], CD160 [15], and/or CD127, which are usually absent on cNK cells.

3.2. ROR $\alpha$. ROR $\alpha$ is highly expressed by ILC2s and is necessary for their development $[32,33]$. ROR $\alpha$ deficiency results in dramatic reduction of ILC2s but does not affect the development of other ILC subsets. The mechanism of 
how ROR $\alpha$ regulates the development of ILC2s is still not clear. ROR $\alpha$ deficiency does not seem to affect Th2 cell differentiation and function. Thus, mice reconstituted with ROR $\alpha$ deficient bone marrow are useful tools for studying ILC2 functions during immune responses.

3.3. Bcl11b. Bcl11b is a critical factor during early stages of T cell development $[34,35]$. But during ILC development, Bcl11b deficiency only blocks the development of ILC2s as shown by two independent studies published this year [36-38]. Bcl11b is expressed as early as the common ILC progenitor stage and it may suppress the development of ILC3s. The mechanism of how Bcll1b specifies ILC2 fate is yet to be determined. Unlike GATA-3 function in mature ILC2s, Bcll1b deletion does not affect the maintenance or function of mature ILC2s despite Bcl11b being highly expressed in mature ILC2s.

3.4. Ahr. The aryl hydrocarbon receptor (Ahr) is well known to be involved in Th17 cell differentiation and function [39]. Ahr is also expressed by both CCR6 $^{+}$and CCR6 ${ }^{-}$ILC3s [40, 41]. In adult $A h r$ deficient mice, ILC3 cell number in the gut is dramatically reduced, probably due to the defective accumulation and/or enhanced apoptosis of ILC3s. Ahr is also critical for the function of ILC3s by regulating IL-22 production and the Ahr effects on ILCs seem to be ILC3 specific.

\section{Progenitors for ILCs}

ILCs, cNK cells, and T cells all develop from CLPs found in fetal liver and adult bone marrow. At late stages of $\mathrm{T}$ cell development, naïve $\mathrm{CD}^{+}$and $\mathrm{CD}^{+} \mathrm{T}$ cells develop from the $\mathrm{CD} 4^{+} \mathrm{CD} 8^{+}$double positive cells in the thymus whereas $\mathrm{CD} 4^{+} \mathrm{T}$ effector Th cells are differentiated from naïve $\mathrm{CD} 4^{+}$ $\mathrm{T}$ cells in the periphery. Considering the functional similarity between mature ILCs and $\mathrm{CD} 4^{+} \mathrm{Th}$ cells, it is reasonable to propose a similar developmental pathway shared by these cells. Thus, a hypothesis concerning ILC development is that, like $\mathrm{T}$ cells, there may be a common progenitor for all innate lymphocytes, including ILCs and cNK cells, after the CLP stage. In a subsequent stage, a common ILC progenitor would be capable of giving rise to all ILCs, in parallel with the potential of naïve $\mathrm{CD}^{+}{ }^{+} \mathrm{T}$ cells to become different Th effector cells. This hypothesis is supported by the fact that certain gene deletions affect the development of all innate lymphocytes and/or all ILCs. Indeed, during the last year, we have witnessed a few breakthrough studies in identifying the common progenitors for ILCs [14, 42].

4.1. Id $2^{+}$ChILP. Id 2 is required for the development of all ILC populations since its deficiency results in the loss of all ILCs. Using an Id 2 reporter mouse strain, several groups have identified and confirmed a common ILC progenitor. A Lineage ${ }^{-} \mathrm{CD} 127^{+}$Flt- $3^{-}$Integrin $\alpha_{4} \beta_{7}{ }^{+}$population has been reported in a stage after CLP and has lost the potential to become T, B, and cNK cells [43]. Id 2 expression within this population has been shown by expression of an Id2 fluorescent reporter. Previously reported immature ILC2s in bone marrow are also $\mathrm{Id} 2^{+}$; however, they can be excluded by CD25 staining. Transfer of these $\mathrm{Id} 2^{+} \mathrm{CD} 25^{-}$progenitor cells gives rise to all "helper" ILCs. The multipotential capacity of these $\mathrm{Id} 2^{+} \mathrm{CD} 25^{-}$progenitors for all ILC subsets was also confirmed by in vitro single cell development assay. These Lineage ${ }^{-} \mathrm{CD} 127^{+}$Flt- $3^{-}$Integrin $\alpha_{4} \beta_{7}{ }^{+} \mathrm{Id} 2^{+} \mathrm{CD} 25^{-}$progenitors are thus termed as "common helper-like innate lymphoid progenitors” (ChILPs).

4.2. GATA-3 Function in Common ILC Progenitors. In addition to the critical role in maintenance and function of ILC2s, GATA-3 is also crucial in the general development of all ILCs. It has been recently reported that Gata3 deficiency prior to the CLP stage affects the development of all ILCs in a cell intrinsic manner, indicating that GATA-3 is a critical regulator for ILC development [31]. In mice carrying a Cre construct driven by the Vav1 promoter to conditionally delete Gata3 at the hematopoietic stem cell stage, dramatic defects in all IL-7R $\alpha$-expressing ILCs in the periphery are noted. Moreover, these Gata3 conditional-deficient mice do not generate lymph nodes or Peyer's patches, consistent with the finding that the development of functional LTi cells is defective at the fetal stage. They also succumb to Citrobacter rodentium infection, consistent with another report showing that GATA3 mediates the development of ILC3s by using chimera mice with hematopoietic precursor cells from E12.5-13.5 $\mathrm{Gata3}^{-/-}$ embryos [44]. The essential role of GATA-3 during ILC development is consistent with its indispensable function during $\mathrm{CD}^{+} \mathrm{T}$ cell development [45] after the $\mathrm{CD} 4^{+} \mathrm{CD} 8^{+}$ stage in the thymus. Consistent with its critical role during ILC development, GATA-3 expression levels in the common ILC progenitors, such as ChILPs, are comparable to that in ILC2s [14]. Thus, GATA-3 is likely a master regulator for the development of common ILC progenitors.

Given that both Id 2 and GATA-3 are highly expressed in ILC progenitors, it is reasonable to speculate that while Id 2 may direct the acquisition of the "innate" feature of ILCs similar to cNK cells GATA-3 may play an indispensable role in directing the "helper" feature of ILCs similar to $\mathrm{CD} 4^{+} \mathrm{T}$ cells. Because of this, GATA-3 may distinguish the "helper" lineage from "cytotoxic" cNK lineage during innate cell development. Altogether, Id 2 and GATA-3 coexpression during the common ILC progenitor stage may establish a special regulatory network that determines the "innate" and "helper" features of the ILCs.

4.3. PLZF ${ }^{+}$Common ILC Progenitor. By analyzing a PLZF fate mapping mouse strain, researchers found that the majority of mature ILCs have expressed PLZF with the exception of the CCR6 ${ }^{+}$ILC3 lineage [42]. PLZF is hardly detectable in mature ILCs but is transiently expressed by a subset of the ChILP population in fetal liver and adult bone marrow [14]. These $\mathrm{PLZF}^{+}$progenitors are multipotential cells and are able to give rise to all ILCs except $\mathrm{CCR}^{+}$LTi cells. Furthermore, these $\mathrm{PLZF}^{+}$progenitors do not develop into $\mathrm{T}$ cells, B cells, or cNK cells. $\mathrm{PLZF}^{+}$progenitors may develop after the ChILP stage and have lost the potential to develop into 
LTi lineage. The function of PLZF during ILC development remains elusive particularly because PLZF is only transiently expressed during the early development stage. Furthermore, although $Z b t b 16^{-1-}$ mice with PLZF deficiency have altered ILC development especially for ILC2s, all the ILCs can still be detected in these mice [42].

An obvious issue for these defined common ILC progenitors is that they are actually heterogeneous populations. Within the ChILP cells, there are $\mathrm{PLZF}^{+}$and $\mathrm{PLZF}^{-}$progenitors. There should be some $\mathrm{CCR}^{+}{ }^{+}$ILC3 progenitors within the $\mathrm{PLZF}^{-}$population, which will never turn on PLZF expression. Even within the $\mathrm{PLZF}^{+}$population, only a minority of the cells have the potential to become multiple ILCs during in vitro single cell development assay. It is likely that both Id $2^{+}$ChILPs and PLZF ${ }^{+}$common ILC progenitors contain a large fraction of partially committed ILC immediate progenitors. The identification of an "authentic" common progenitor multipotent for all ILCs is still required. Meanwhile, in addition to fetal liver and adult bone marrow, the common ILC progenitors may also exist in other tissues, such as fetal intestine, as was shown in a recent study using arginase-1 reporter mice [46]. This report suggests that we should not limit ourselves to study fetal liver and adult bone marrow cells to gain further knowledge on ILC progenitors.

4.4. Immediate ILC Subset Precursors. As mentioned above, many ILC progenitors found in fetal liver and adult bone marrow may have already committed partially to a specific ILC lineage. Indeed, Bcll1b-expressing progenitors appear within the ChILP population; yet these cells have already committed to the ILC2 fate [37]. There are also abundant immature ILC2s, presumably the most immediate ILC2 precursors, present in the bone marrow; these cells express mature ILC2 markers Sca-1 and CD25 [30]. Immature ILC1s also exist in bone marrow termed as Eomes ${ }^{-} \mathrm{CD} 49 \mathrm{a}^{+} \mathrm{NK} 1.1^{+} \mathrm{NKp} 46^{+}[14]$. PLZF fate mapping analysis indicates that most immature ILCls have expressed PLZF [47] and thus are derived from the PLZF ${ }^{+}$ILC progenitors rather than the cNK progenitors.

4.5. Common Progenitors for ILCs and cNK Cells. A couple of other transcription factors, Nfil3 [48-51] and TOX [52], have also been found to be involved in the development of both ILCs and cNK cells. Since their expression is detected earlier than Id 2 expression, which occurs at the ChILP stage, the expression of these transcription factors may mark even earlier common progenitors for both ILCs and cNK cells, similar to $\mathrm{CD}^{+}{ }^{+} \mathrm{CD} 8^{+}$thymocytes during $\mathrm{T}$ cell development. However, the phenotypes of ILC development in mice deficient in either of these regulators are not as dramatic as those resulting from $I d 2$ or Gata 3 deficiency. The mechanisms through which Nfil3 and TOX function during ILC and cNK cell development require further investigation.

Given the functional similarities between the innate ILCs and cNK cells and the adaptive CD4 and CD8 T cells, it is reasonable to propose that ILCs and cNK cells follow a similar developmental pathway to that of T cells. A CLP may give rise to a common progenitor for all innate lymphocytes including ILCs and cNK cells, which subsequently gives rise to ChILPs.
Distinct ILC subsets are further developed from the PLZF ChILP stage as the differentiation of Th effectors from naïve $\mathrm{CD}^{+}{ }^{+} \mathrm{T}$ cells. Thus, the development of innate lymphocyte subsets, including ILCs and cNK cells, to a certain extent, mirrors that of $\mathrm{T}$ cells (Figure 1).

\section{Regulatory Network in Common ILC Progenitor}

As discussed above, more and more regulators are being found to be involved in the development of ILCs. Interestingly, most of these regulators are also involved with $\mathrm{T}$ cell development. However, distinct from $\mathrm{T}$ cell development, the regulatory functions of the various transcription factors during ILC development are not related to TCR-mediated thymic selection, which, to some extent, may explain why the deficiency of certain regulators has distinct effects on ILC and T cell development. Although deletion of either one of the newly identified genes, including Nfil3, TOX, or Tcf7 $[53,54]$, results in defective ILC development to a various extent, none of these defects are as severe as that resulting from Id2 or Gata3 deficiency. Thus, it is possible that in the early progenitors of the ILC lineage, these regulators may function through a network designed for fine-tuning ILC development. Id2 and GATA-3 might form the core complex, while other regulators are involved in tuning the optimal function of the core components. Id 2 or Gata3 deficiency dramatically affects ILC development, whereas deletion of other regulatory components results in various incomplete defects of ILC development.

5.1. Nfil3. Nuclear factor interleukin-3 (Nfil3; also known as E4-binding protein 4, E4BP4), a basic region leucine zipper transcription factor, is critical for cNK cell development [55]. However, Nfil3 deficiency also affects ILC development [48-51]. Based on this, it is possible that Nfil3 is expressed and functions at a progenitor stage common to both ILCs and cNK cells. Nfil3 deficient mice showed dramatic defects during the transition from CLP to ChILP stage [48, 51]. However, ILC development is not completely abolished in Nfil3 deficient mice; in the periphery, there are still substantial numbers of ILCs that have escaped the developmental block, although the population is too small to control infectious challenges. Nfil3 expression is induced by IL-7 signaling, which is also critical for the development of ILCs. Nfil3 may work by regulating TOX [50] and Id2 [51] expression. After development, Nfil3 maintains a very high level of expression in mature ILCs, a level higher than that in cNK cells. Nfil3 is dispensable for ILC3 maintenance; however, its function in other mature ILCs remains unclear.

5.2. TOX. Thymocyte selection-associated high-mobility group box protein (TOX) is a member of the high-mobility group box superfamily. It contains a DNA-binding domain and is required for the development of T [56, 57], NK [58], NKT [59], and LTi cells [58]. A new study this year showed that TOX also has broad effects in ILC development [52]. In Tox deficient mice, the ChILPs as well as mature ILCs were 


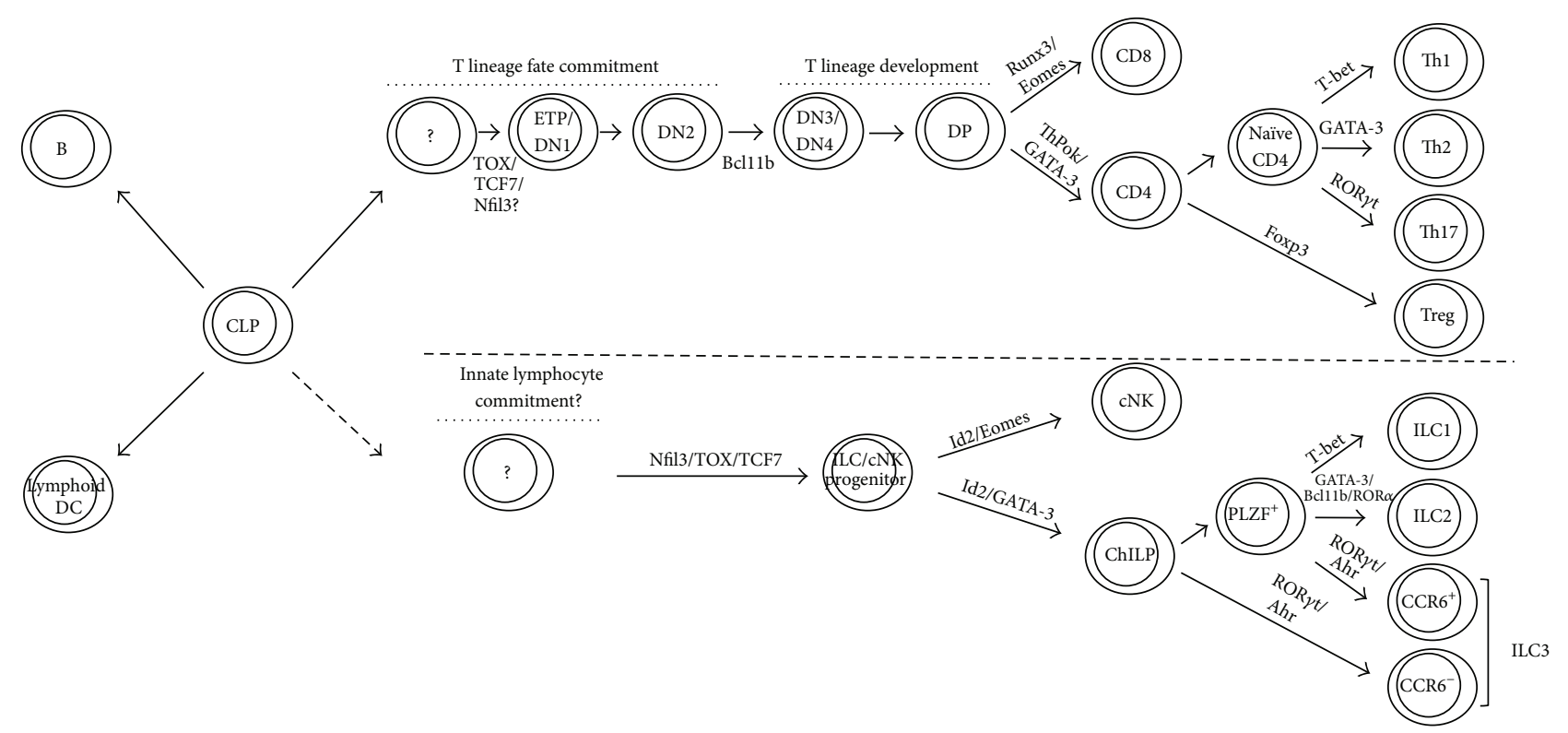

FIGURE 1: Parallels between the development of innate lymphocytes and T cells. Lymphoid DCs, B cells, T cells, and innate lymphocytes including ILCs and cNK cells are derived from common lymphoid progenitors (CLPs). While innate lymphocytes mainly develop in the bone marrow, $\mathrm{T}$ cells develop in the thymus. T cell development passes through a fate determination (from ETP/DN1 to DN2 to DN3) stage before $\mathrm{CD}^{+}$and $\mathrm{CD}^{+} \mathrm{T}$ cells develop separately from progenitor-like CD4 $4^{+} \mathrm{CD} 8^{+} \mathrm{DP}$ cells. After $\mathrm{CD} 4^{+} \mathrm{T}$ cell development, effector Th cells are differentiated from naïve $\mathrm{CD} 4^{+} \mathrm{T}$ cells upon activation. In parallel, for the innate lymphocyte development, there may also be a stage when the innate fate is determined followed by the generation of ILC and cNK common progenitors. ILC and cNK lineage then develop separately under the guidance of distinct master regulators. Similar to effector Th cells differentiating from naïve CD $4^{+} \mathrm{T}$ cells, all mature ILC subsets also develop from a common progenitor. Therefore, ILCs share with T cells in utilizing multiple transcription factors at similar stages during their development/differentiation.

severely reduced compared with these cells in wild type mice. There is an intrinsic defect in the Notch signaling pathway in Tox deficient ChILPs [52], which may explain the defect in ILC development in Tox deficient mice.

5.3. TCF-1. T cell factor 1 (TCF-1) is a critical transcription factor for $\mathrm{T}$ cell fate specification at the early development stage [60]. Tcf7 (gene encode TCF-1) deficiency affects both ILCs $[53,54]$ and cNK cells [61]. Similar as during T cell development, TCF-1 is regulated by Notch signaling and may be involved in the induction of IL-7R and GATA-3 expression during ILC development $[53,54]$.

5.4. Putative Regulatory Network in ILC Progenitors. Based on the sequential expression of Nfil3, TOX, TCF-1, Id2, and GATA-3 and the knowledge we have concerning their relationships in different systems, it is likely that Nfil3 expression initially increases after the CLP stage, which in turn regulates the expression of TOX and Id2. After Id2 is turned on, the core assembly involved in directing the "innate" features of ILC development begins to function. Although GATA-3 is expressed at low levels at the CLP stage, its function during ILC development requires high expression levels. TOX is involved in the regulation of Notch signaling pathway, which in turn regulates TCF-1 and GATA-3. TCF1 is then required for optimal expression of GATA-3. Upon increased expression of GATA-3, the "helper" transcription factor assembly would execute the gene expression program needed for ILC function. In this way, Nfil3, TOX, and TCF1 , in connection with the Notch pathways, form a network to prepare for the upregulation of Id2 and GATA-3, which together form the executive regulatory network to direct lineage fate determination of ILCs, possibly with continued assistance from the initiation transcription factors such as Tcf7, Tox, and Nfil3 (Figure 2). Additional experiments are required to confirm this proposed regulatory network model and it is likely that additional regulators for ILC development may soon be discovered.

\section{Conclusions}

Studies on the newly identified ILCs in recent years have enriched our knowledge on the innate lymphoid lineage development and have provided us with more evidence supporting the close relationship between the innate and adaptive immune systems during evolution. The classification of the ILC population was initially based on their capacity to produce a similar set of effector cytokines compared to Th cells. Subsequent studies have revealed that the development of ILCs is also regulated by a similar set of key transcription factors required for T cell development. After the CLP stage, innate lymphoid lineages and $\mathrm{T}$ cells lineages start to develop separately but the regulatory mechanisms may still be shared. Nfil3, TOX, and TCF-1 expression at an early stage immediately after CLP in the innate lymphoid lineage may mark the 


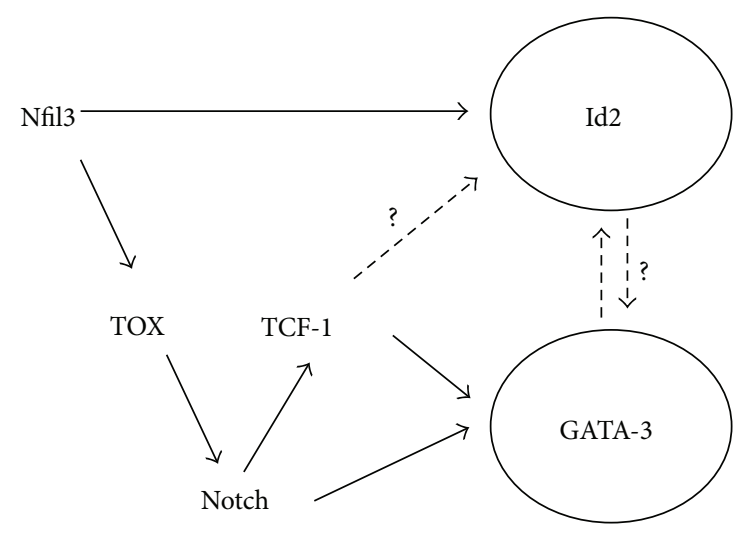

FIgURE 2: A potential transcriptional regulatory network determining the generation of a common ILC progenitor. Critical transcription factors during common ILC progenitor development are expressed at various stages. After the CLP stage, Nfil3 expression increases followed by Id 2 and TOX induction. TOX then induces Notch, which is required for TCF-1 and GATA-3 upregulation. TCF1 further enhances GATA-3 expression. Although it has not been reported, TCF-1 may also play a role in enhancing Id 2 expression. The regulatory network eventually results in optimal Id 2 and GATA3 expression in the common ILC progenitors. Once Id 2 and GATA-3 expression reaches a threshold, they orchestrate the acquisition of the "innate" and "helper" features of the ILCs, possibly also with assistance from other transcription factors.

common progenitor for both ILCs and cNK cells. ILC and $\mathrm{cNK}$ lineages are further separated by the induced expression of Id 2 and GATA-3 in the common ILC progenitors. At later stages, additional regulators are upregulated to drive the distinct fates of different ILC subsets. In conclusion, the ILC cell fate is precisely regulated during development by a network of multiple serially expressed regulators, which may form a regulatory network during development and render the developed ILCs with both "innate" and "helper" features.

\section{Conflict of Interests}

The authors declare that there is no conflict of interests regarding the publication of this paper.

\section{Acknowledgments}

The authors thank Dr. Darah Christie for her comments and editing the paper. The work is supported by the Intramural Research Program of National Institute of Health (NIH), National Institute of Allergy and Infectious Diseases (NIAID), NIH, USA.

\section{References}

[1] H. Spits, D. Artis, M. Colonna et al., "Innate lymphoid cells-a proposal for uniform nomenclature," Nature Reviews Immunology, vol. 13, no. 2, pp. 145-149, 2013.

[2] H. Spits and J. P. Di Santo, "The expanding family of innate lymphoid cells: regulators and effectors of immunity and tissue remodeling," Nature Immunology, vol. 12, no. 1, pp. 21-27, 2011.
[3] R. E. Mebius, P. Rennert, and I. L. Weissman, "Developing lymph nodes collect $\mathrm{CD}^{+} \mathrm{CD}^{-} \mathrm{LT} \beta^{+}$cells that can differentiate to APC, NK cells, and follicular cells but not T or B cells," Immunity, vol. 7, no. 4, pp. 493-504, 1997.

[4] K. A. Kelly and R. Scollay, "Seeding of neonatal lymph nodes by $\mathrm{T}$ cells and identification of a novel population of CD3-CD4 $4^{+}$ cells," European Journal of Immunology, vol. 22, no. 2, pp. 329334, 1992.

[5] K. Moro, T. Yamada, M. Tanabe et al., "Innate production of $\mathrm{T}(\mathrm{H}) 2$ cytokines by adipose tissue-associated c-Kit(+)Sca-1(+) lymphoid cells," Nature, vol. 463, no. 7280, pp. 540-544, 2010.

[6] D. R. Neill, S. H. Wong, A. Bellosi et al., "Nuocytes represent a new innate effector leukocyte that mediates type-2 immunity," Nature, vol. 464, no. 7293, pp. 1367-1370, 2010.

[7] A. E. Price, H.-E. Liang, B. M. Sullivan et al., "Systemically dispersed innate IL-13-expressing cells in type 2 immunity," Proceedings of the National Academy of Sciences of the United States of America, vol. 107, no. 25, pp. 11489-11494, 2010.

[8] N. Satoh-Takayama, C. A. J. Vosshenrich, S. Lesjean-Pottier et al., "Microbial flora drives interleukin 22 production in intestinal $\mathrm{NKp}^{6+}$ cells that provide innate mucosal immune defense," Immunity, vol. 29, no. 6, pp. 958-970, 2008.

[9] S. Buonocore, P. P. Ahern, H. H. Uhlig et al., "Innate lymphoid cells drive interleukin-23-dependent innate intestinal pathology," Nature, vol. 464, no. 7293, pp. 1371-1375, 2010.

[10] G. F. Sonnenberg, L. A. Monticelli, M. M. Elloso, L. A. Fouser, and D. Artis, "CD4 ${ }^{+}$lymphoid tissue-inducer cells promote innate immunity in the gut," Immunity, vol. 34, no. 1, pp. 122134, 2011.

[11] C. S. N. Klose, E. A. Kiss, V. Schwierzeck et al., "A T-bet gradient controls the fate and function of CCR6-RORgammat+ innate lymphoid cells," Nature, vol. 494, no. 7436, pp. 261-265, 2013.

[12] L. C. Rankin, J. R. Groom, M. Chopin et al., “The transcription factor T-bet is essential for the development of NKp $46^{+}$innate lymphocytes via the Notch pathway," Nature Immunology, vol. 14, no. 4, pp. 389-395, 2013.

[13] G. Sciumè, K. Hirahara, H. Takahashi et al., "Distinct requirements for T-bet in gut innate lymphoid cells," The Journal of Experimental Medicine, vol. 209, no. 13, pp. 2331-2338, 2012.

[14] C. S. N. Klose, M. Flach, L. Möhle et al., "Differentiation of type 1 ILCs from a common progenitor to all helper-like innate lymphoid cell lineages," Cell, vol. 157, no. 2, pp. 340-356, 2014.

[15] A. Fuchs, W. Vermi, J. S. Lee et al., "Intraepithelial type 1 innate lymphoid cells are a unique subset of IL-12- and IL-15responsive IFN-gamma-producing cells," Immunity, vol. 38, no. 4, pp. 769-781, 2013.

[16] A. Diefenbach, M. Colonna, and S. Koyasu, "Development, differentiation, and diversity of innate lymphoid cells," Immunity, vol. 41, no. 3, pp. 354-365, 2014.

[17] K. Yasuda, T. Muto, T. Kawagoe et al., "Contribution of IL-33-activated type II innate lymphoid cells to pulmonary eosinophilia in intestinal nematode-infected mice," Proceedings of the National Academy of Sciences of the United States of America, vol. 109, no. 9, pp. 3451-3456, 2012.

[18] J. C. Nussbaum, S. J. van Dyken, J. von Moltke et al., "Type 2 innate lymphoid cells control eosinophil homeostasis," Nature, vol. 502, no. 7470, pp. 245-248, 2013.

[19] G. F. Sonnenberg, L. A. Fouser, and D. Artis, "Border patrol: regulation of immunity, inflammation and tissue homeostasis at barrier surfaces by IL-22," Nature Immunology, vol. 12, no. 5, pp. 383-390, 2011. 
[20] D. K. Sojka, B. Plougastel-Douglas, L. Yang et al., “Tissueresident natural killer (NK) cells are cell lineages distinct from thymic and conventional splenic NK cells," eLife, vol. 2014, no. 3, Article ID e01659, 2014.

[21] T. Y. F. Halim, C. A. Steer, L. Mathä et al., "Group 2 innate lymphoid cells are critical for the initiation of adaptive T helper 2 cell-mediated allergic lung inflammation," Immunity, vol. 40, no. 3, pp. 425-435, 2014.

[22] C. J. Oliphant, Y. Y. Hwang, J. A. Walker et al., "MHCIImediated dialog between group 2 innate lymphoid cells and $\mathrm{CD}^{+} \mathrm{T}$ cells potentiates type 2 immunity and promotes parasitic helminth expulsion," Immunity, vol. 41, no. 2, pp. 283295,2014

[23] M. R. Hepworth, L. A. Monticelli, T. C. Fung et al., "Innate lymphoid cells regulate $\mathrm{CD} 4^{+}$T-cell responses to intestinal commensal bacteria," Nature, vol. 498, no. 7452, pp. 113-117, 2013.

[24] M. R. Hepworth, T. C. Fung, S. H. Masur et al., "Group 3 innate lymphoid cells mediate intestinal selection of commensal bacteria-specific $\mathrm{CD}^{+} \mathrm{T}$ cells," Science, vol. 348 , no. 6238, pp. 1031-1035, 2015.

[25] L. A. Monticelli, G. F. Sonnenberg, M. C. Abt et al., "Innate lymphoid cells promote lung-tissue homeostasis after infection with influenza virus," Nature Immunology, vol. 12, no. 11, pp. 1045-1054, 2011.

[26] J. R. Brestoff, B. S. Kim, S. A. Saenz et al., "Group 2 innate lymphoid cells promote beiging of white adipose tissue and limit obesity," Nature, vol. 519, pp. 242-246, 2014.

[27] M.-W. Lee, J. I. Odegaard, L. Mukundan et al., "Activated type 2 innate lymphoid cells regulate beige fat biogenesis," Cell, vol. 160, no. 1-2, pp. 74-87, 2015.

[28] Y. Goto, T. Obata, J. Kunisawa et al., "Innate lymphoid cells regulate intestinal epithelial cell glycosylation," Science, vol. 345, no. 6202, Article ID 1254009, 2014.

[29] J. M. Pickard, C. F. Maurice, M. A. Kinnebrew et al., "Rapid fucosylation of intestinal epithelium sustains host-commensal symbiosis in sickness," Nature, vol. 514, no. 7524, pp. 638-641, 2014.

[30] T. Hoyler, C. S. N. Klose, A. Souabni et al., "The transcription factor GATA-3 controls cell fate and maintenance of type 2 innate lymphoid cells," Immunity, vol. 37, no. 4, pp. 634-648, 2012.

[31] R. Yagi, C. Zhong, D. L. Northrup et al., "The transcription factor GATA3 is critical for the development of all IL-7R $\alpha$ expressing innate lymphoid cells," Immunity, vol. 40, no. 3, pp. 378-388, 2014.

[32] S. H. Wong, J. A. Walker, H. E. Jolin et al., "Transcription factor ROR $\alpha$ is critical for nuocyte development," Nature Immunology, vol. 13, no. 3, pp. 229-236, 2012.

[33] T. Y. F. Halim, A. MacLaren, M. T. Romanish, M. J. Gold, K. M. McNagny, and F. Takei, "Retinoic-acid-receptor-related orphan nuclear receptor alpha is required for natural helper cell development and allergic inflammation," Immunity, vol. 37, no. 3, pp. 463-474, 2012.

[34] T. Ikawa, S. Hirose, K. Masuda et al., "An essential developmental checkpoint for production of the T cell lineage," Science, vol. 329, no. 5987, pp. 93-96, 2010.

[35] P. Li, S. Burke, J. Wang et al., "Reprogramming of T cells to natural killer-like cells upon Bcl11b deletion," Science, vol. 329, no. 5987, pp. 85-89, 2010.
[36] J. A. Walker, C. J. Oliphant, A. Englezakis et al., "Bcl11b is essential for group 2 innate lymphoid cell development," The Journal of Experimental Medicine, vol. 212, no. 6, pp. 875-882, 2015.

[37] Y. Yu, C. Wang, S. Clare et al., “The transcription factor Bcl11b is specifically expressed in group 2 innate lymphoid cells and is essential for their development," The Journal of Experimental Medicine, vol. 212, no. 6, pp. 865-874, 2015.

[38] C. Zhong and J. Zhu, "Bcl11b drives the birth of ILC2 innate lymphocytes," The Journal of Experimental Medicine, vol. 212, no. 6, p. 828, 2015.

[39] F. J. Quintana, A. S. Basso, A. H. Iglesias et al., "Control of $\mathrm{T}_{\text {reg }}$ and $\mathrm{T}_{H} 17$ cell differentiation by the aryl hydrocarbon receptor," Nature, vol. 453, no. 7191, pp. 65-71, 2008.

[40] E. A. Kiss, C. Vonarbourg, S. Kopfmann et al., "Natural aryl hydrocarbon receptor ligands control organogenesis of intestinal lymphoid follicles," Science, vol. 334, no. 6062, pp. 1561-1565, 2011.

[41] J. Qiu, J. J. Heller, X. Guo et al., "The aryl hydrocarbon receptor regulates gut immunity through modulation of innate lymphoid cells," Immunity, vol. 36, no. 1, pp. 92-104, 2012.

[42] M. G. Constantinides, B. D. McDonald, P. A. Verhoef, and A. Bendelac, "A committed precursor to innate lymphoid cells," Nature, vol. 508, no. 7496, pp. 397-401, 2014.

[43] C. Possot, S. Schmutz, S. Chea et al., "Notch signaling is necessary for adult, but not fetal, development of RORgammat(+) innate lymphoid cells," Nature Immunology, vol. 12, no. 10, pp. 949-958, 2011.

[44] N. Serafini, R. G. J. Klein Wolterink, N. Satoh-Takayama et al., "RORgammat+ group 3 innate lymphoid cells," The Journal of Experimental Medicine, vol. 211, no. 2, pp. 199-208, 2014.

[45] I.-C. Ho, T.-S. Tai, and S.-Y. Pai, "GATA3 and the T-cell lineage: essential functions before and after T-helper-2-cell differentiation," Nature Reviews Immunology, vol. 9, no. 2, pp. $125-135,2009$.

[46] J. K. Bando, H.-E. Liang, and R. M. Locksley, "Identification and distribution of developing innate lymphoid cells in the fetal mouse intestine," Nature Immunology, vol. 16, no. 2, pp. 153-160, 2015.

[47] M. G. Constantinides, H. Gudjonson, B. D. McDonald et al., "PLZF expression maps the early stages of ILC1 lineage development," Proceedings of the National Academy of Sciences of the United States of America, vol. 112, no. 16, pp. 5123-5128, 2015.

[48] T. L. Geiger, M. C. Abt, G. Gasteiger et al., "Nfil3 is crucial for development of innate lymphoid cells and host protection against intestinal pathogens," The Journal of Experimental Medicine, vol. 211, no. 9, pp. 1723-1731, 2014.

[49] C. Seillet, L. C. Rankin, J. R. Groom et al., "Nfil3 is required for the development of all innate lymphoid cell subsets," The Journal of Experimental Medicine, vol. 211, no. 9, pp. 1733-1740, 2014.

[50] X. Yu, Y. Wang, M. Deng et al., "The basic leucine zipper transcription factor NFIL3 directs the development of a common innate lymphoid cell precursor," eLife, vol. 3, 2014.

[51] W. Xu, R. Domingues, D. Fonseca-Pereira et al., "NFIL3 orchestrates the emergence of common helper innate lymphoid cell precursors," Cell Reports, vol. 10, no. 12, pp. 2043-2054, 2015.

[52] C. R. Seehus, P. Aliahmad, B. de la Torre et al., "The development of innate lymphoid cells requires TOX-dependent generation of a common innate lymphoid cell progenitor," Nature Immunology, vol. 16, no. 6, pp. 599-608, 2015. 
[53] L. A. Mielke, J. R. Groom, L. C. Rankin et al., "TCF-1 controls ILC2 and NKp46+RORgammat+ innate lymphocyte differentiation and protection in intestinal inflammation," The Journal of Immunology, vol. 191, no. 8, pp. 4383-4391, 2013.

[54] Q. Yang, L. A. Monticelli, S. A. Saenz et al., "T cell factor 1 is required for group 2 innate lymphoid cell generation," Immunity, vol. 38, no. 4, pp. 694-704, 2013.

[55] S. Kamizono, G. S. Duncan, M. G. Seidel et al., "Nfil3/E4bp4 is required for the development and maturation of NK cells in vivo," The Journal of Experimental Medicine, vol. 206, no. 13, pp. 2977-2986, 2009.

[56] B. Wilkinson, J. Y.-F. Chen, P. Han, K. M. Rufner, O. D. Goularte, and J. Kaye, "TOX: an HMG box protein implicated in the regulation of thymocyte selection," Nature Immunology, vol. 3, no. 3, pp. 272-280, 2002.

[57] P. Aliahmad, E. O’Flaherty, P. Han et al., "TOX provides a link between calcineurin activation and CD8 lineage commitment," The Journal of Experimental Medicine, vol. 199, no. 8, pp. 10891099, 2004.

[58] P. Aliahmad, B. de la Torre, and J. Kaye, "Shared dependence on the DNA-binding factor TOX for the development of lymphoid tissue-inducer cell and NK cell lineages," Nature Immunology, vol. 11, no. 10, pp. 945-952, 2010.

[59] P. Aliahmad and J. Kaye, "Development of all CD4 T lineages requires nuclear factor TOX," The Journal of Experimental Medicine, vol. 205, no. 1, pp. 245-256, 2008.

[60] B. N. Weber, A. W.-S. Chi, A. Chavez et al., "A critical role for TCF-1 in T-lineage specification and differentiation," Nature, vol. 476, no. 7358, pp. 63-69, 2011.

[61] W. Held, H. Clevers, and R. Grosschedl, "Redundant functions of TCF-1 and LEF-1 during T and NK cell development, but unique role of TCF-1 for Ly 49 NK cell receptor acquisition," European Journal of Immunology, vol. 33, no. 5, pp. 1393-1398, 2003. 


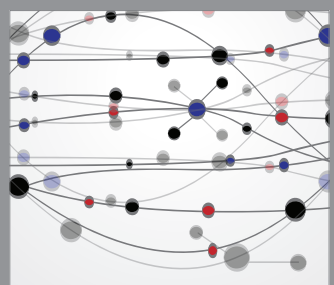

The Scientific World Journal
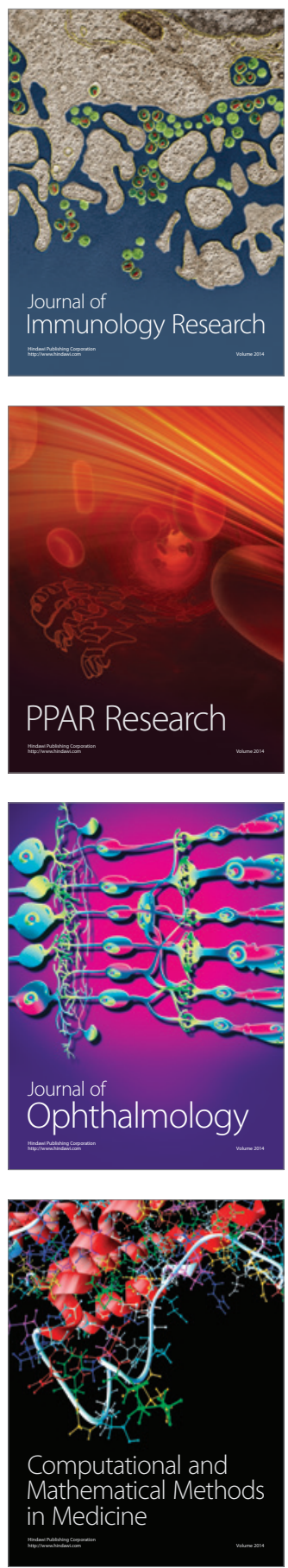

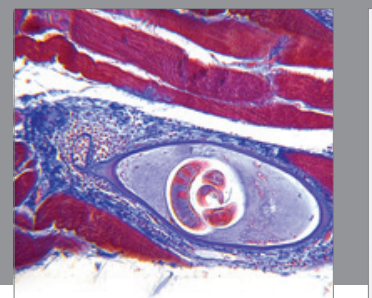

Gastroenterology

Research and Practice
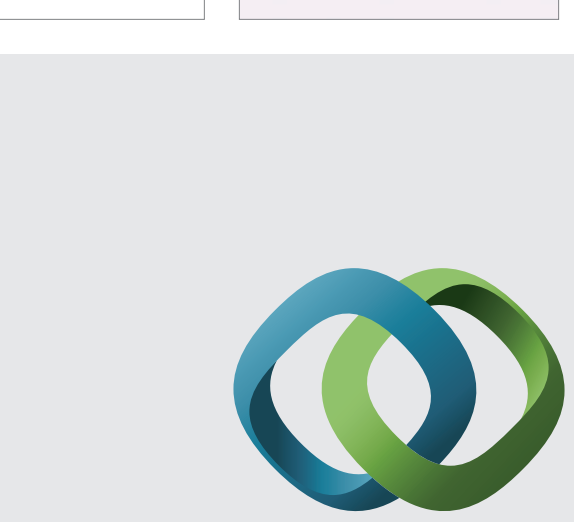

\section{Hindawi}

Submit your manuscripts at

http://www.hindawi.com
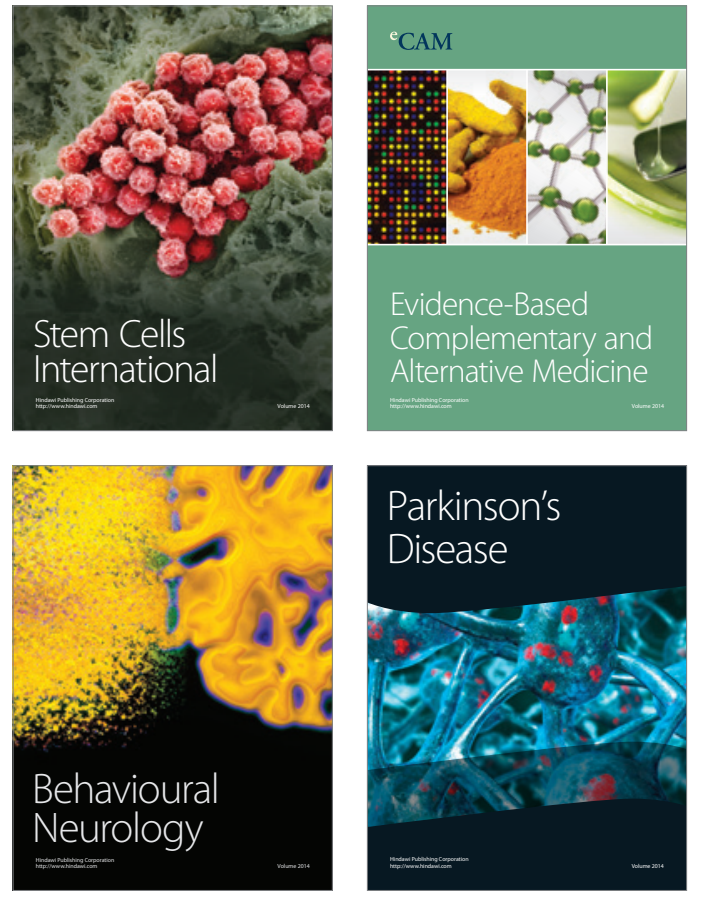
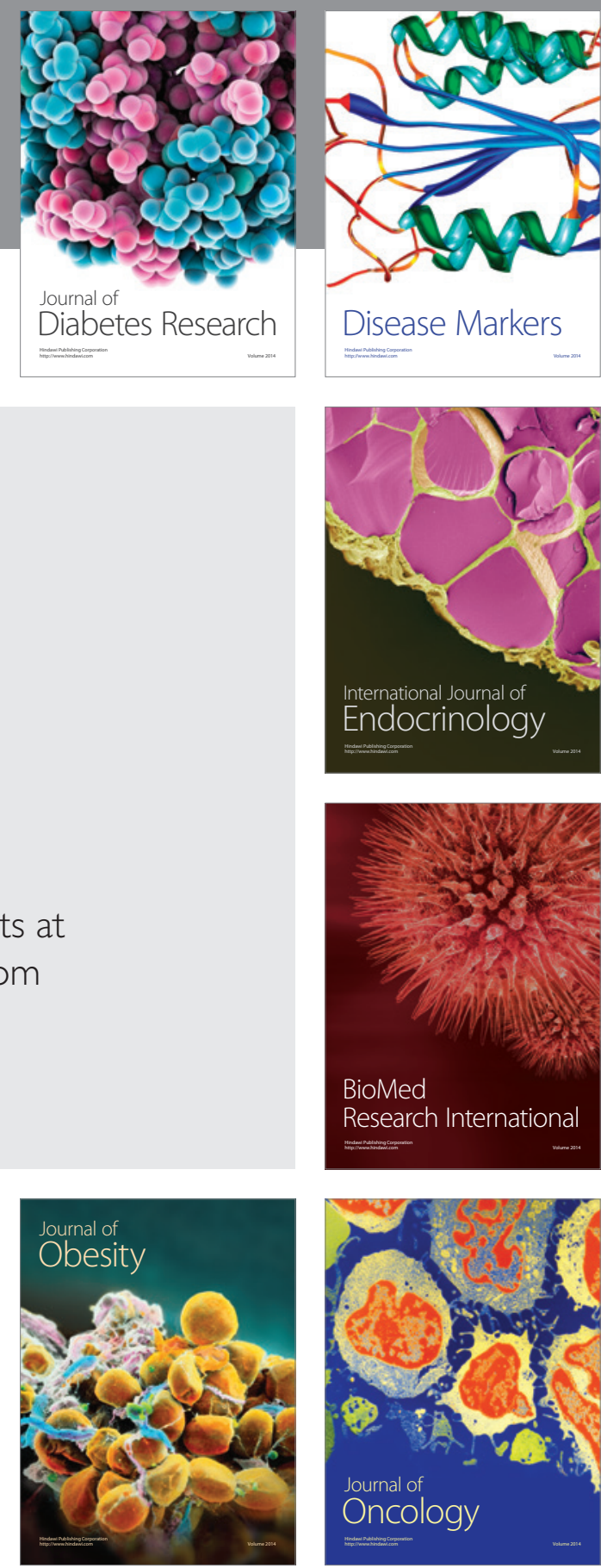

Disease Markers
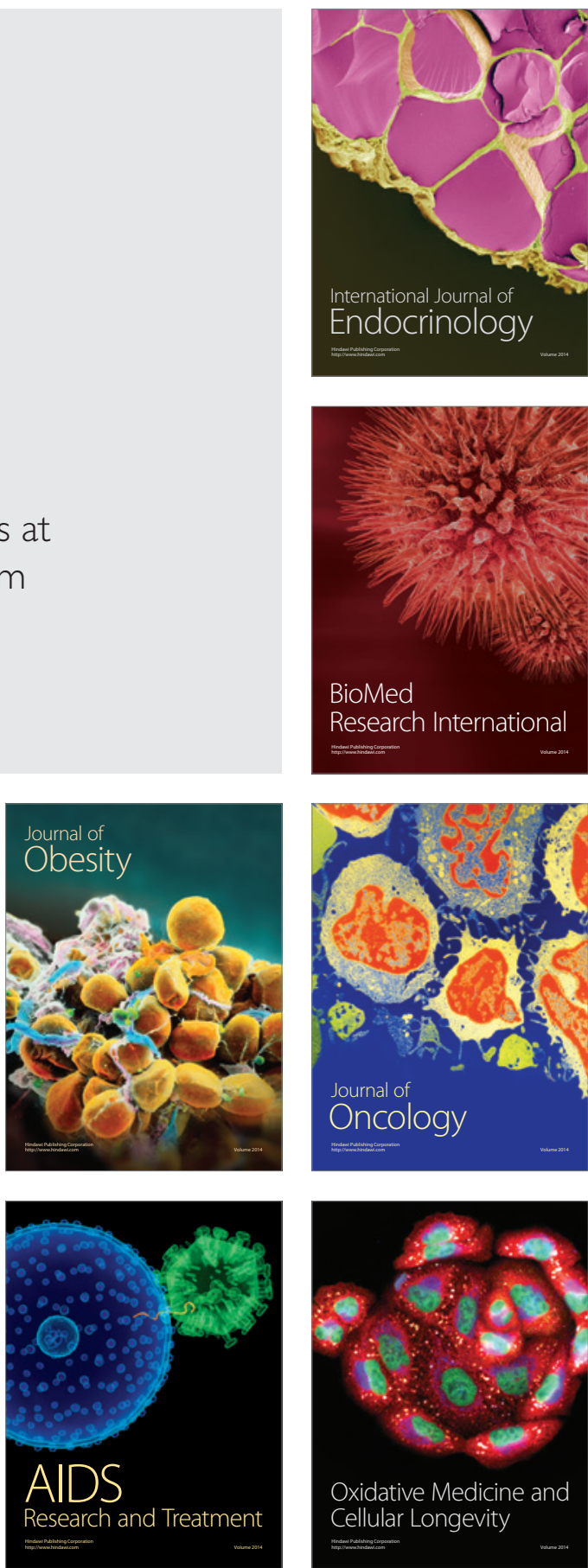